\title{
Reducing Signaling Overhead for Femtocell/Macrocell Networks
}

\author{
Huai-Lei Fu, Student Member, IEEE, Phone Lin, Senior Member, IEEE, \\ Yi-Bing Lin, Fellow, IEEE
}

\begin{abstract}
Femtocell technology has been proposed to offload user data traffic from the macrocell to the femtocell and extend the limited coverage of the macrocell in Mobile Communications Networks (MCNs). In existing commercial femtocell/macrocell networks, a widely accepted solution to configure the Location Areas (LAs) is to partition the femtocells overlapped with a macrocell into small groups and to assign each group with a unique LA ID different from that of the macrocell. Such configuration can reduce the paging cost in the mobility management, but increases registration signaling overhead due to discontinuous coverage of femtocells. To reduce signaling overhead in the femtocell/macrocell network, we propose a Delay Registration (DR) algorithm that postpones the registration until the delay timer expires when the MS moves into the overlapped femtocell. Analytical models and simulation experiments are proposed to investigate the performance of the DR algorithm. Our analytical models are general enough to accommodate various MS mobility behaviors. Our study can provide guidelines for the operators to set up a delay timer to reduce signaling overhead while sustaining the traffic offloading capability of the femtocell.
\end{abstract}

\section{Index Terms}

Femtocell, Macrocell, Mobile Communications Networks, Mobility Management

\section{INTRODUCTION}

In a Mobile Communications Network $(\mathrm{MCN})$, the service area is populated with Base Stations (BSs). The radio coverage of a BS (or a sector of the BS) is called a cell. In an outdoor environment, network operators deploy macrocells with radio coverage area of 0.5 to 2 kilometer-radius. The fast growing population of mobile users leads to an exponential increase in user data traffic demand for the $\mathrm{MCN}$, but the capacity of outdoor macrocells are not sufficient to satisfy such demand.

The femtocell [1-3] (also called home BS) with low deployment cost was proposed to offload the user data traffic to the macrocell and extend the limited coverage of the macrocell.

H.-L. Fu is with the Department of Computer Science and Information Engineering, National Taiwan University, Taipei, Taiwan R.O.C. (e-mail: vicfu@pcs.csie.ntu.edu.tw).

P. Lin is the contact author and with the Department of Computer Science and Information Engineering, National Taiwan University, Taipei, Taiwan R.O.C. (e-mail: plin@csie.ntu.edu.tw).

Y.-B. Lin is with Department of Computer Science and Information Engineering, National Chiao Tung University, Hsinchu, Taiwan R.O.C. (e-mail: liny@csie.nctu.edu.tw). 


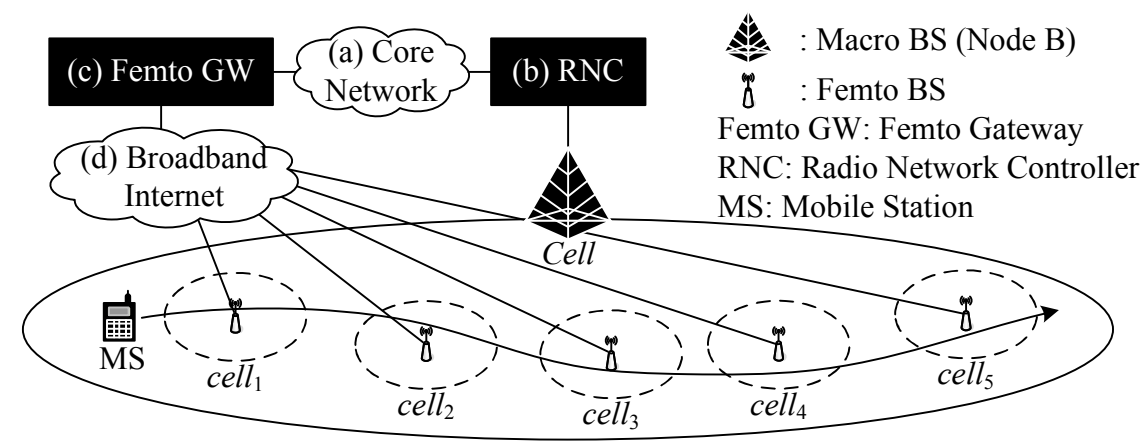

Fig. 1. An example of the femtocell/macrocell network architecture

A femtocell is the radio coverage of a short-range, low-cost, and low-power wireless BS, typically covering an area with the radius of 5 to 20 meters [2]. Femtocells operate in the same licensed spectrum as a macrocell. In femtocell and macrocell deployment environment planned by some mobile network operators, hundreds to thousands of femtocells are overlapped with a macrocell.

In the view point of mobile network operators, traffic offloading is one of the major reasons for femtocell deployment. When a Mobile Station (MS) resides in a femtocell overlapped with a macrocell, as long as the MS has the authority to access the femtocell, the MS prefers to connect to the femtocell so as to offload traffic to the macrocell.

We name the MCNs with femtocells and macrocells as femtocell/macrocell networks. Figure 1 depicts an example of the femtocell/macrocell network based on the Universal Mobile Telecommunications System (UMTS) [4]. In this figure, the macrocell Cell (see the solid circle) overlays with the femtocells $\mathrm{cell}_{1}, \ldots$, cell $_{5}$ (see the dashed circles). The macrocell connects to the Core Network (CN; Figure 1 (a)) through the Radio Network Controller (RNC; Figure 1 (b)). The femtocells connect to the $\mathrm{CN}$ through the Femto Gateway (Femto GW; Figure 1 (c)) and a broadband Internet network [5] (Figure 1 (d)). The Femto GW plays the role of an RNC. Importantly, the service area of femtocells may be discontinued. For example, in Figure 1, cell $1, \ldots$, cell $_{5}$ can be treated as "islands" that do not overlap with each other.

The cells in the femtocell/macrocell network are grouped into Location Areas (LAs). Each 
LA is assigned with a unique LA ID (LAI). The LAs are used for mobility management. The mobility management consists of "registration" and "call termination". Each macrocell or femtocell uses a wireless broadcast channel to broadcast its individually corresponding LAI. The MS listens to the wireless broadcast channel to identify in which LA it resides. When the MS moves from one LA to another (that is, the LAI stored in the MS storage is different from the received LAI), the MS initiates a "registration" to report the LAI of the cell in which it resides to the location database in the $\mathrm{CN}$. This process is also known as "location update". "Call termination" refers to the process of routing an incoming call to an MS. In this process, the CN obtains the LA of the MS by querying the location database, instructs all cells in the LA to "page" the MS, and then sets up the call to the MS through the cell in which the MS responds to the "page". Details of registration and call termination can be found in [6].

There are two alternatives to assign LAs in the femtocell/macrocell network [7], [8]. In the first alternative, all femtocells overlapped with a macrocell are assigned to the same LA as that of the macrocell. If more than hundreds of femtocells are overlapped with the macrocell, all of the femtocells and the macrocell must together page an MS for its incoming call, cumulating high paging cost. In the second alternative, the femtocells overlapped with the macrocell are partitioned into small groups, and each group is assigned with a unique LAI different from that of the macrocell.

Compared with the first alternative, the second alternative significantly reduces the paging cost [8]. Hence, the second alternative is more often exercised in existing commercial mobile communications networks. However, a potential problem of this second alternative is that registration occurs every time the MS moves between the macrocell and the overlapped femtocell, increasing signaling overhead to the network. Take for example the network architecture in Figure 1, where the LA of the macrocell Cell is $L_{m}$, and $L_{f}$ is the LA assigned to the overlapped femtocells $\mathrm{cell}_{1}, \mathrm{cell}_{2}, \ldots, \mathrm{cell}_{5}$. Since the service area of these femtocells is discontinuous, when an MS moves from one femtocell to another, it crosses through the 
macrocell and switches between $L_{m}$ and $L_{f}$, triggering registration. For example, the MS

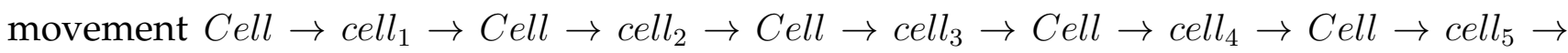
Cell results in as many as ten registrations.

In this paper, we focus on the location update issue in the femtocell/macrocell networks. To be more specific, we investigate the mobility management for the second alternative of LA layout. The major challenge is that the frequent execution of registration due to small and discontinuous femtocell coverage causes high signaling overhead. Note that some mobile network operators exercise the business models such that private home BSs can be purchased by users, and each home BS can only be accessed by a particular user's mobile devices. In this scenario, not many subsequent registrations will be sent to many femtocells, so the frequent registration issue does not exist.

Following most of location update studies [9-11], we do not consider channel capacity of femtocells or macrocells, which is an independent issue dealt by admission control. The channel capacity issue is out of the scope of this paper and should be treated separately to investigate original call admission and handoff strategies for femtocell/macrocell networks.

Previous works [12-14] have addressed the mobility management issue for the macrocellmicrocell hierarchical mobile networks. Both [12] and [13] addressed the mobility management issue for the macrocell-microcell hierarchical mobile networks in which microcell radio coverage is continuous, i.e., when an MS moves from one microcell to another, it does not cross any overlapped macrocell. On the other hand, our work addresses the issue caused by coverage discontinuity of femtocells. In [12], the authors proposed a macro-micro paging scheme (based on the predefined delay constraints) to balance the paging load between the macrocell and overlapped microcell. They did not focus on the signaling overhead reduction for registration (i.e., location update), and therefore the proposed technique does not reduce registration overhead. In [13], the authors proposed a cross-tier registration and paging scheme. In this scheme, a registration is executed only when an MS crosses the boundary between two LAs in the macrocell tier. Based on the paging load, the CN 
determines whether to page an MS through the macrocell tier or microcell tier. The proposed scheme requires significant modification on the existing mobility management protocols at the core network side, which is considered impractical in the femtocell/macrocell networks. Work [14] proposed the integration of high-tier mobile networks and low-tier mobile networks, and these networks operate in separate mobility management protocols. Work [14] also proposed intelligent algorithms to determine whether the MS should perform the registration operation when the MS switches tiers. The architecture considered in [14] is more complicated than the femtocell/macrocell networks, and the performance is not as good as our approach due to an extra-layer of mobility management integration of two networks.

In this paper, we propose the Delay Registration (DR) algorithm that postpones registration when an MS moves into the overlapped femtocell in the femtocell/macrocell network. Details of the DR algorithm is given in the next section. We propose analytical models and conduct simulation experiments to investigate the performance of the DR algorithm. In the proposed analytical models, we assume general distributions for the residence time periods in the overlapped femtocell and in the non-overlapped areas of the macrocell. Our analytical model is general enough to accommodate various MS mobility behaviors. The analytical model is validated against the simulation experiments.

The remaining parts of the paper are organized as follows. Details of the DR algorithm are given in Section 2. In Section 3, we describe the analytical models. In Section 4, we evaluate the performance of the DR algorithm and provide guidelines for the setup of the delay timer. Section 5 concludes this paper.

\section{The Delay Registration Algorithm}

This section proposes the Delay Registration (DR) algorithm. The DR algorithm is exercised at the MS to determine whether the registration should be performed. No modification is required at the network side. 
To simplify our description, we consider the following MS moving behavior: A macrocell overlays with several femtocells. Let $L_{m}$ be the LAI assigned to the macrocell and $L_{f}$ be the LAI assigned to the overlapped femtocells. Initially, an MS is in the non-overlapped area in the macrocell, and $L_{m}$ is the LAI stored in the location database. At time $t$, the MS moves into the overlapped femtocell (i.e., the MS can also receive $L_{f}$ ) and stays in the overlapped femtocell for $t_{f}$. At $t+t_{f}$, the MS moves from the overlapped femtocell into the macrocell, and can no longer receive $L_{f}$.

In the standard 3GPP algorithms for mobility management, at $t$ one registration is executed to change $L_{m}$ to $L_{f}$ in the location database, and at $t+t_{f}$ another registration is performed to change $L_{f}$ to $L_{m}$. Here two registrations are performed. Note that if the MS just passes by the femtocell, that is, $t_{f}$ is as short as only a few seconds, we describe the MS movement as transient. If transient occurs, it is most likely that no call behavior (call origination/termination) will take place during $\left[t, t+t_{f}\right]$. Thus these two registrations during the transient period may be avoided without significantly affecting traffic offloading capability of the femtocell. To avoid registrations during the transient period, we propose the DR algorithm that introduces a delay timer to postpone the registration until the timer expires.

At $t$, the DR algorithm suspends the registration and starts the delay timer with length $t_{d}$. Two cases are considered:

- If $t_{d}<t_{f}$, as the delay timer expires at $t+t_{d}$, the MS is still in the overlapped femtocell. The MS stops the delay timer and initiates the registration to change $L_{m}$ to $L_{f}$.

- Otherwise (i.e., $t_{d} \geq t_{f}$ ), the MS stops the delay timer at $t+t_{f}$, and no registration occurs during $\left[t, t+t_{f}\right]$.

During the period $\left[t, t+\min \left(t_{d}, t_{f}\right)\right]$, if a call requests arrives, the request is potentially handled through the macrocell instead of through the overlapped femtocell, and the traffic may not be offloaded from the macrocell to the femtocell. Clearly, the longer the delay timer, the more signaling overhead (caused by registration) avoided. But meanwhile, it is more 
TABLE 1

Comparison between the 3GPP algorithms and the DR algorithm

w/o femtocell 3GPP algorithms DR algorithm

\begin{tabular}{llll}
\hline \hline Signaling overhead & N/A & high & low \\
\hline Offload traffic & N/A & high & medium $\sim$ high \\
\hline
\end{tabular}

likely that the traffic to the macrocell can not be offloaded. In the next section, we propose analytical models to study the tradeoff between signaling overhead reduction and traffic offloading capability.

To summarize, in Table 1, we compare the signaling overhead and traffic offloading capability of the MCN without femtocells, femtocell/macrocell networks with the standard 3GPP algorithms, and femtocell/macrocell networks with the DR algorithm. The MCN without femtocells does not have traffic offloading capability nor the signaling overhead. The standard 3GPP has the high signaling overhead and high traffic offloading capability. On the other hand, the DR algorithm has the low signaling overhead, but medium to high traffic offloading capability.

The impacts of the DR algorithm on user experience, device energy consumption, and mobile network operators are discussed as follows: With the DR algorithm, by properly setting the delay timer, the signaling overhead is reduced, and the traffic offloading capability of femtocells can still be achieved. In the femtocell/macrocell networks, more bandwidth is available, and the user will have better experience in call admission. For energy consumption, the DR algorithm reduces the number of registrations executed by an MS, thus saves the MS power consumption. For mobile network operators (or carriers), with the DR algorithm, less registrations (i.e., location database updates) will save more network bandwidth and switch capacity. Note that in most designs of core networks, as indicated in [15], $15 \%$ of computing power is allocated for location update. Intensive registration due to switching among femtocells and macrocells may exceed $15 \%$ of computing power and degrade the performance for the core network. Our DR algorithm reduces significant 
registration overhead in femtocell/macrocell networks.

\section{AnAlytical Models}

In this section, we propose analytical models to study the impacts of the setup of the delay timer on performance tradeoff between the signaling overhead and traffic offloading capability. Our study provides guidelines for selecting an appropriate $t_{d}$ such that the signaling overhead decreases, and the traffic offloading capability can still be achieved. The performance metrics derived in our analytical model includes two output measures signaling overhead ratio $r\left(t_{d}\right)$ and potential offload traffic ratio $\rho\left(t_{d}\right)$ :

Signaling overhead ratio $r\left(t_{d}\right)$ : Define a "crossing" as the event when the MS moves from the macrocell to the overlapped femtocell or vice versa. Consider the inter-call arrival time $t_{c}$ between two consecutive call request arrivals to the MS. Let $N_{c}$ be the number of crossings the MS has during $t_{c}$, and $N_{r}$ be the number of registrations executed by the MS during $t_{c}$. Obviously, $N_{c} \geq N_{r}$. If $t_{d}=0$ (i.e., the standard 3GPP algorithms are exercised), then $N_{r}=N_{c}$. We denote $E\left[N_{r}\left(t_{d}\right) \mid N_{c} \geq 1\right]$ for the DR algorithm with delay $t_{d}$, and $E\left[N_{r}(0) \mid N_{c} \geq 1\right]$ for the 3GPP algorithms. We define $r\left(t_{d}\right)$ as

$$
r\left(t_{d}\right)=\frac{E\left[N_{r}\left(t_{d}\right) \mid N_{c} \geq 1\right]}{E\left[N_{r}(0) \mid N_{c} \geq 1\right]}=\frac{E\left[N_{r}\left(t_{d}\right)\right] / \operatorname{Pr}\left[N_{c} \geq 1\right]}{E\left[N_{r}(0)\right] / \operatorname{Pr}\left[N_{c} \geq 1\right]}=\frac{E\left[N_{r}\left(t_{d}\right)\right]}{E\left[N_{r}(0)\right]},
$$

where $0<r\left(t_{d}\right) \leq 1$. A smaller $r\left(t_{d}\right)$ implies that more signaling overhead for the DR algorithm is reduced.

Potential offload traffic ratio $\rho\left(t_{d}\right)$ : Let $p\left(t_{d}\right)$ and $p(0)$ denote the probabilities that a call request arrives when the MS registers to the femtocell (i.e., the location information for the MS is the LAI of a femtocell) for the DR algorithm (with delay period $t_{d}$ ) and for the 3GPP algorithms, respectively. With the DR algorithm, the traffic offloading capability degrades due to the delay timer, i.e., less call request arrivals when the MS registers to the femtocell than that with the 3GPP algorithms. Hence we have $p\left(t_{d}\right) \leq p(0)$. Define $\rho\left(t_{d}\right)$ as

$$
\rho\left(t_{d}\right)=\frac{p\left(t_{d}\right)}{p(0)}
$$


where $0<\rho\left(t_{d}\right) \leq 1$. A larger $\rho\left(t_{d}\right)$ implies that the DR algorithm causes less degradation for the traffic offloading capability of the femtocell.

We model the behavior of an MS in the femtocell/macrocell network as follows. Consider the timing diagram in Figure 2. Without loss of generality, the macrocell belongs to location area $L_{m}$, and the femtocells belongs to $L_{f}$. The radio coverage areas of femtocells in $L_{f}$ are discontinuous. When the MS passes through these discontinuous femtocells, it switches between $L_{m}$ and $L_{f}$. More specifically, the MS stays in $L_{f}$ for a period $t_{f}$ and then moves to $L_{m}$ for another period $t_{m}$.

During the timer period $t_{c}$ between two consecutive call request arrivals, for $i \geq 1$, let $t_{m, i}$ be the $i$ th time that the MS visits $L_{m}$ after the previous call request arrival, and $t_{f, i}$ be the $i$ th time that the MS visits $L_{f}$ after the previous call request arrival. By convention, when $i=0, t_{m, i}\left(t_{f, i}\right)$ refers to the time period when the previous call arrives in a macrocell (in a femtocell). In the DR algorithm, each $t_{f, i}$ is associated with the delay timer with length $t_{d}$. In the analytical model, we make the following three assumptions:

A1. The call request arrivals to an MS form a Poisson process with rate $\lambda$, i.e., the intercall arrival time $t_{c}$ is exponentially distributed with the density function $f(t)=\lambda e^{-\lambda t}$. The Poisson process assumption is widely used in teletraffic analysis to model the call request arrival behavior [16], [17].

A2. The $t_{m, i}$ and $t_{f, i}$ random variables are i.i.d. with the general density function $f_{m}(t)$ and $f_{f}(t)$, the mean $1 / \eta_{m}$ and $1 / \eta_{f}$, and the Laplace transform $f_{m}^{*}(s)$ and $f_{f}^{*}(s)$, respectively.

A3. The $t_{d}$ random variable is exponentially distributed with the mean $1 / \theta$ and the density function $f_{d}(t)=\theta e^{-\theta t}$. From implementation perspectives, fixed $t_{d}$ seems to be a convenient choice. The complexities of generating fixed $t_{d}$ and exponential $t_{d}$ are basically the same in terms of implementation cost [18]. We will extend exponential $t_{d}$ to fixed $t_{d}$ in simulation experiments.

During $t_{c}$, let $t_{c, i}$ be the time period between when the MS visits $L_{f}$ for the $i$ th time and when the next call request arrives. The density function of $t_{c, i}$ is denoted by $f_{c, i}(t)$. Since 
$t_{c}$ is exponentially distributed, from the PASTA property of Poisson processes [19], $t_{c, i}$ has the same distribution as $t_{c}$, i.e., the density function $f_{c, i}(t)=\lambda e^{-\lambda t}$. Figure 2 illustrates four scenarios that may occur during period $t_{c}$. In Figure $2(\mathrm{a})$, the previous call request arrives when the MS is in $L_{m}$, and the next call request arrives when the MS is in $L_{f}$. In Figure 2 (b), both call requests arrive at $L_{m}$. In Figure 2 (c), both call requests arrive at $L_{f}$. In Figure 2 (d), the previous call request arrives at $L_{f}$, and the next call request arrives at $L_{m}$.

The notations used in the analytical models are summarized as follows:

- $t_{c}$ : the time period between two consecutive call request arrivals to the MS. The density function of $t_{c}$ is $f(t)=\lambda e^{-\lambda t}$.

- $t_{c, i}:$ the time period between when the MS visits $L_{f}$ for the $i$ th time and when the next call request arrives. The density function of $t_{c, i}$ is $f_{c, i}(t)=\lambda e^{-\lambda t}$.

- $t_{d}$ : the delay period. The density function of $t_{d}$ is $f_{d}(t)=\theta e^{-\theta t}$.

- $t_{f, i}$ : the $i$ th time that the MS visits $L_{f}$ after the previous call request arrival (i.e., the MS is in the overlapped femtocell). The density function, mean and Laplace transform of $t_{f, i}$ are $f_{f}(t), 1 / \eta_{f}$ and $f_{f}^{*}(s)$, respectively.

- $t_{m, i}$ : the $i$ th time that the MS visits $L_{m}$ after the previous call request arrival (i.e., the MS is in the non-overlapped area of the macrocell). The density function, mean and Laplace transform of $t_{m, i}$ are $f_{m}(t), 1 / \eta_{m}$ and $f_{m}^{*}(s)$, respectively.

- $N_{c}$ : the number of crossings for the MS during $t_{c}$.

- $N_{r}$ : the number of registrations executed by the MS during $t_{c}$.

- $r\left(t_{d}\right)$ : the signaling overhead ratio for the DR algorithm.

- $\rho\left(t_{d}\right)$ : the potential offload traffic ratio for the DR algorithm.

- $\tau_{m}$ : the time period between when the previous call request arrives at $L_{m}$ and when the MS visits $L_{f}$ for the first time.

\subsection{Derivation of Signaling Overhead Ratio $r\left(t_{d}\right)$}

To derive the $r\left(t_{d}\right)$ performance, we first derive $E\left[N_{r}(0)\right]$ and then $E\left[N_{r}\left(t_{d}\right)\right]$, respectively described in Section 3.1.1 and Section 3.1.2. Applying the derivation results, (17) and (30), 


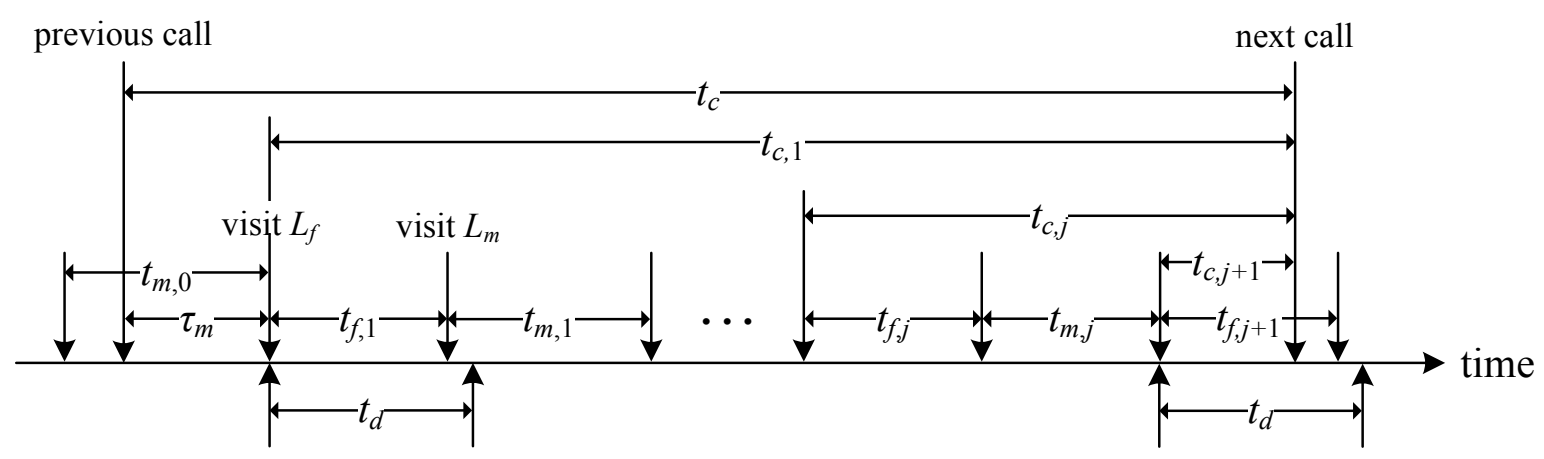

(a) Case Ia: the previous call arrives when the MS is in $L_{m}$, and the next call arrives when the MS is in $L_{f}$.

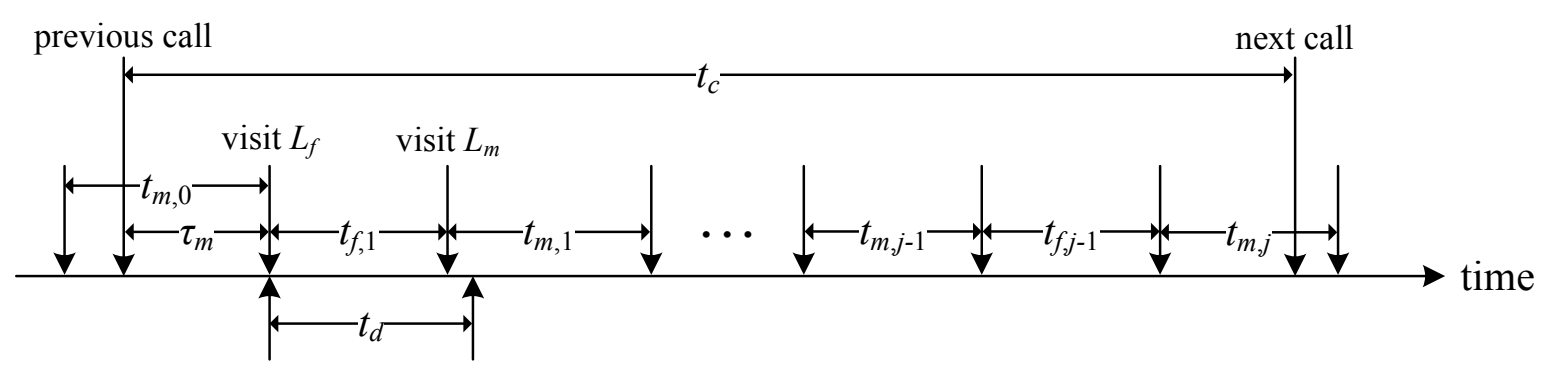

(b) Case Ib: the previous call arrives when the MS is in $L_{m}$, and the next call arrives when the MS is in $L_{m}$.

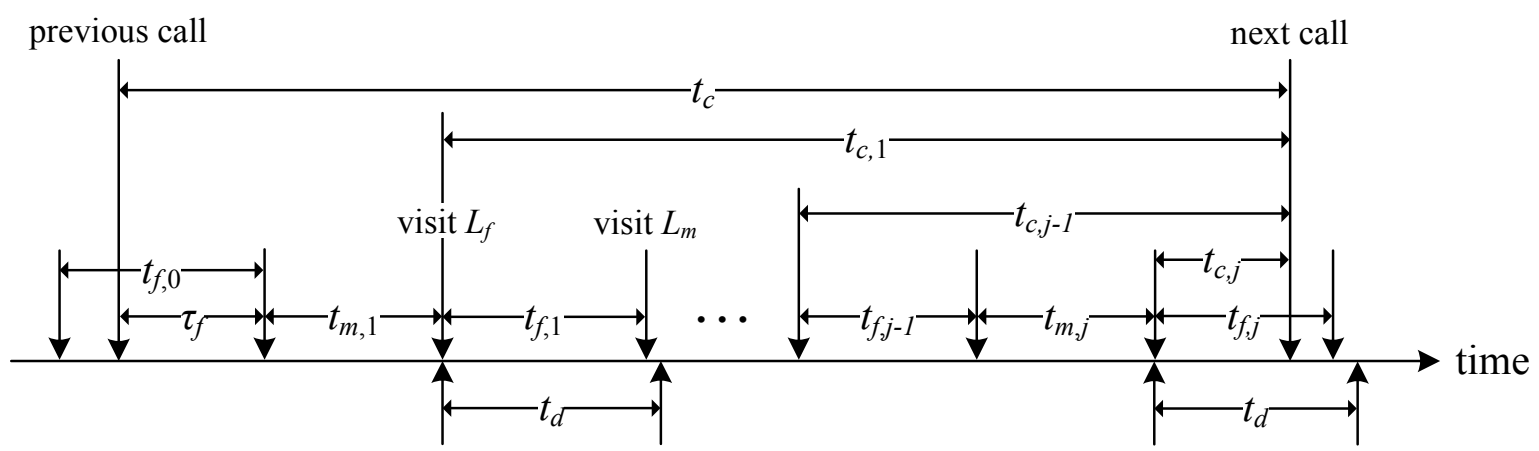

(c) Case IIa: the previous call arrives when the MS is in $L_{f}$, and the next call arrives when the MS is in $L_{f}$.

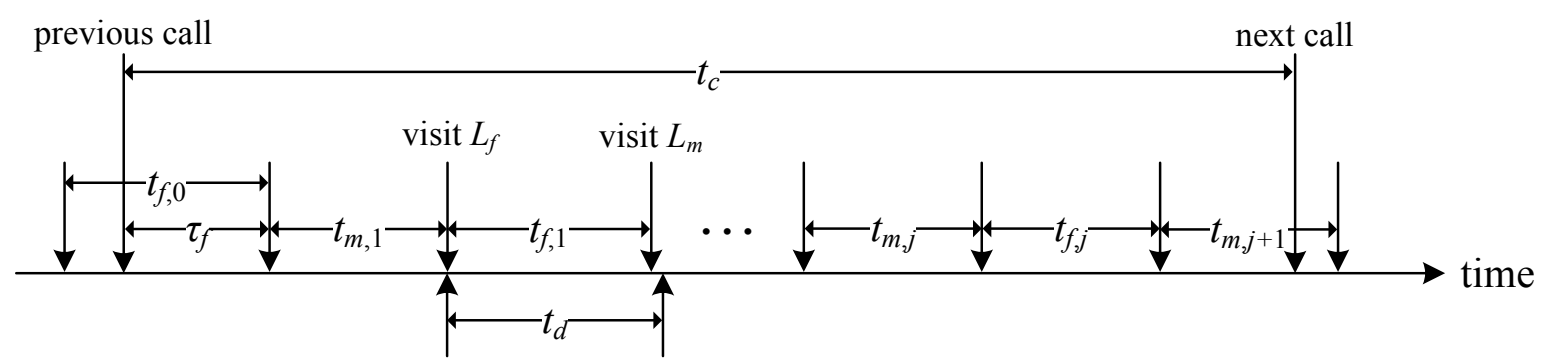

(d) Case IIb: the previous call arrives when the MS is in $L_{f}$, and the next call arrives when the MS is in $L_{m}$.

Fig. 2. The timing diagrams for MS movement and call activities 
into (1), we have

$$
r\left(t_{d}\right)=\frac{\theta\left[1-f_{f}^{*}(\lambda+\theta)\right]}{\lambda+\theta} .
$$

\subsubsection{Derivation of $E\left[N_{r}(0)\right]$}

If $t_{d}=0$ (i.e., the 3GPP algorithms are exercised), the MS immediately executes a registration when it moves from $L_{m}$ to $L_{f}$ or moves from $L_{f}$ to $L_{m}$. Then we have

$$
E\left[N_{r}(0)\right]=E\left[N_{c}\right]
$$

We consider two cases to derive $\operatorname{Pr}\left[N_{c}=k\right]$ for $k \geq 1$ :

Case I: The previous call arrives when the MS is in $L_{m}$. See Figure 2 (a) and (b).

Case II: The previous call arrives when the MS is in $L_{f}$. See Figure 2 (c) and (d).

Let $N_{I, c}$ and $N_{I I, c}$ be the number $N_{c}$ conditioning on Case I and Case II, respectively. We express $\operatorname{Pr}\left[N_{c}=k\right]$ by

$$
\operatorname{Pr}\left[N_{c}=k\right]=\operatorname{Pr}\left[N_{I, c}=k\right] \operatorname{Pr}[\text { Case I }]+\operatorname{Pr}\left[N_{I I, c}=k\right] \operatorname{Pr}[\text { Case II }] .
$$

As shown in Figure 2, the timing diagram for the MS alters between $t_{m, i}$ and $t_{f, i}$. According to the alternating renewal process [20], we have

$$
\operatorname{Pr}[\text { Case I }]=\frac{E\left[t_{m, i}\right]}{E\left[t_{m, i}\right]+E\left[t_{f, i}\right]}=\frac{\eta_{f}}{\eta_{m}+\eta_{f}},
$$

and

$$
\operatorname{Pr}[\text { Case II }]=\frac{\eta_{m}}{\eta_{m}+\eta_{f}} .
$$

We apply (5) and (6) into (4) to yield

$$
\operatorname{Pr}\left[N_{c}=k\right]=\frac{\eta_{f} \operatorname{Pr}\left[N_{I, c}=k\right]+\eta_{m} \operatorname{Pr}\left[N_{I I, c}=k\right]}{\eta_{m}+\eta_{f}},
$$

where $\operatorname{Pr}\left[N_{I, c}=k\right]$ and $\operatorname{Pr}\left[N_{I I, c}=k\right]$ are derived as follows:

The derivation of $\operatorname{Pr}\left[N_{I, c}=k\right]$ : Consider Case I in Figure 2 (a) and (b). Let $\tau_{m}$ be the time period between when the previous call request arrives at $L_{m}$ and when the MS visits $L_{f}$ for the first time, i.e., $\tau_{m}$ is the residual life of $t_{m, 0}$. Let $r_{m}(t)$ be the density function 
of $\tau_{m}$. From the residual life theorem [20] and the previous work [10], the Laplace transform of $r_{m}(t)$ is obtained by

$$
r_{m}^{*}(s)=\left(\frac{\eta_{m}}{s}\right)\left[1-f_{m}^{*}(s)\right]
$$

Let $t_{M, k}=\tau_{m}+\sum_{i=1}^{k}\left(t_{f, i}+t_{m, i}\right)$ and $t_{F, k}=\tau_{m}+t_{f, 1}+\sum_{i=1}^{k}\left(t_{m, i}+t_{f, i+1}\right)$. By convention, $t_{M, 0}=\tau_{m}$ and $t_{F, 0}=\tau_{m}+t_{f, 1}$. Assume that $t_{M, k}$ and $t_{F, k}$ have the density functions $f_{M, j}(t)$ and $f_{F, j}(t)$ with the Laplace transforms $f_{M, k}^{*}(s)$ and $f_{F, k}^{*}(s)$, respectively. From the convolution rule of Laplace transform, we have

$$
f_{M, k}^{*}(s)=r_{m}^{*}(s)\left[f_{m}^{*}(s) f_{f}^{*}(s)\right]^{k},
$$

and

$$
f_{F, k}^{*}(s)=r_{m}^{*}(s) f_{f}^{*}(s)\left[f_{m}^{*}(s) f_{f}^{*}(s)\right]^{k} .
$$

In the following, we consider two cases, Case Ia (i.e., the next call request arrives when the MS is in $L_{f}$ ) and Case Ib (i.e., the next call request arrives when the MS is in $L_{m}$ ) to derive $\operatorname{Pr}\left[N_{I, c}=k\right]$.

Case Ia: See Figure 2 (a). The previous call request arrives during $t_{m, 0}$, and the next call request arrives during $t_{f, j+1}$ where $j \geq 0$. In this case, $t_{M, j}<t_{c}<t_{F, j}$, and $k$ must be an odd number, i.e., $k=2 j+1$. Then we have

$$
\begin{aligned}
\operatorname{Pr}\left[N_{I, c}=2 j+1\right] & =\operatorname{Pr}\left[t_{M, j}<t_{c}<t_{F, j}\right]=\operatorname{Pr}\left[t_{c}>t_{M, j}\right]-\operatorname{Pr}\left[t_{c}>t_{F, j}\right] \\
& =\int_{t_{c}=0}^{\infty} \int_{t_{M, j}=0}^{t_{c}} f_{M, j}\left(t_{M, j}\right) \lambda e^{-\lambda t_{c}} d t_{M, j} d t_{c} \\
& -\int_{t_{c}=0}^{\infty} \int_{t_{F, j}=0}^{t_{c}} f_{F, j}\left(t_{F, j}\right) \lambda e^{-\lambda t_{c}} d t_{F, j} d t_{c} \\
& =f_{M, j}^{*}(\lambda)-f_{F, j}^{*}(\lambda) .
\end{aligned}
$$

Apply (8), (9) and (10) into (11) to have

$$
\operatorname{Pr}\left[N_{I, c}=2 j+1\right]=\left(\frac{\eta_{m}}{\lambda}\right)\left[1-f_{m}^{*}(\lambda)\right]\left[1-f_{f}^{*}(\lambda)\right]\left[f_{m}^{*}(\lambda) f_{f}^{*}(\lambda)\right]^{j} .
$$


Case Ib: See Figure 2 (b). The previous call request arrives during $t_{m, 0}$, and the next call request arrives during $t_{m, j}$ where $j \geq 1$. In this case, $t_{F, j-1}<t_{c}<t_{M, j}$, and $k$ must be an even number, i.e., $k=2 j$. Similar to the derivation of (12), we have

$$
\begin{aligned}
\operatorname{Pr}\left[N_{I, c}=2 j\right] & =\operatorname{Pr}\left[t_{F, j-1}<t_{c}<t_{M, j}\right] \\
& =\left(\frac{\eta_{m}}{\lambda}\right) f_{f}^{*}(\lambda)\left[1-f_{m}^{*}(\lambda)\right]^{2}\left[f_{m}^{*}(\lambda) f_{f}^{*}(\lambda)\right]^{j-1}
\end{aligned}
$$

The derivation of $\operatorname{Pr}\left[N_{I I, c}=k\right]$ : Consider Case II in Figure 2 (c) and (d). The derivation for $\operatorname{Pr}\left[N_{I I, c}=k\right]$ is similar to that for $\operatorname{Pr}\left[N_{I, c}=k\right]$ except that in Case II, the previous call request arrives during $t_{f, 0}$ and then the MS alters between the time period pairs $\left(t_{f}, t_{m}\right)$. Thus we have

$$
\operatorname{Pr}\left[N_{I I, c}=2 j\right]=\left(\frac{\eta_{f}}{\lambda}\right) f_{m}^{*}(\lambda)\left[1-f_{f}^{*}(\lambda)\right]^{2}\left[f_{m}^{*}(\lambda) f_{f}^{*}(\lambda)\right]^{j-1},
$$

and

$$
\operatorname{Pr}\left[N_{I I, c}=2 j+1\right]=\left(\frac{\eta_{f}}{\lambda}\right)\left[1-f_{f}^{*}(\lambda)\right]\left[1-f_{m}^{*}(\lambda)\right]\left[f_{m}^{*}(\lambda) f_{f}^{*}(\lambda)\right]^{j}
$$

Apply (12), (13), (14) and (15) into (7), and for $k \geq 1$, we have

$$
\operatorname{Pr}\left[N_{c}=k\right]= \begin{cases}\frac{2 \eta_{m} \eta_{f}\left[1-f_{m}^{*}(\lambda)\right]\left[1-f_{f}^{*}(\lambda)\right]\left[f_{m}^{*}(\lambda) f_{f}^{*}(\lambda)\right]^{\frac{k-1}{2}}}{\lambda\left(\eta_{m}+\eta_{f}\right)}, & \text { odd } k ; \\ \frac{\eta_{m} \eta_{f}\left\{f_{f}^{*}(\lambda)\left[1-f_{m}^{*}(\lambda)\right]^{2}+f_{m}^{*}(\lambda)\left[1-f_{f}^{*}(\lambda)\right]^{2}\right\}\left[f_{m}^{*}(\lambda) f_{f}^{*}(\lambda)\right]^{\frac{k}{2}-1}}{\lambda\left(\eta_{m}+\eta_{f}\right)}, & \text { even } k .\end{cases}
$$

Then from (16), we have

$$
E\left[N_{r}(0)\right]=E\left[N_{c}\right]=\sum_{k=1}^{\infty} k \operatorname{Pr}\left[N_{c}=k\right]=\frac{2 \eta_{m} \eta_{f}}{\lambda\left(\eta_{m}+\eta_{f}\right)}
$$

\subsubsection{Derivation of $E\left[N_{r}\left(t_{d}\right)\right]$}

Consider the time period $t_{f, i}$ for $i \geq 1$. Let $\alpha$ be the probability that the MS executes the registration during $t_{f, i}$ (i.e., $t_{d}<t_{f, i}$ ) under the condition that the next call request does not 
arrive during $t_{f, i}$, that is,

$$
\begin{aligned}
\alpha & =\operatorname{Pr}\left[t_{d}<t_{f, i} \mid t_{c, i}>t_{f, i}\right]=\frac{\operatorname{Pr}\left[t_{d}<t_{f, i}<t_{c, i}\right]}{\operatorname{Pr}\left[t_{c, i}>t_{f, i}\right]} \\
& =\frac{\int_{t_{d}=0}^{\infty} \int_{t_{f, i}=t_{d}}^{\infty} \int_{t_{c, i}=t_{f, i}}^{\infty} \lambda e^{-\lambda t_{c, i}} f_{f}\left(t_{f, i}\right) \theta e^{-\theta t_{d}} d t_{c, i} d t_{f, i} d t_{d}}{\int_{t_{f, i}=0}^{\infty} \int_{t_{c, i}=t_{f, i}}^{\infty} \lambda e^{-\lambda t_{c, i}} f_{f}\left(t_{f, i}\right) d t_{c, i} d t_{f, i}} \\
& =\frac{f_{f}^{*}(\lambda)-f_{f}^{*}(\lambda+\theta)}{f_{f}^{*}(\lambda)} .
\end{aligned}
$$

Let $\beta$ be the probability that the MS executes the registration before the next call request arrives under the condition that the next call request arrives during $t_{f, i}$, and we have

$$
\begin{aligned}
\beta & =\operatorname{Pr}\left[t_{d}<t_{c, i} \mid t_{c, i}<t_{f, i}\right]=\frac{\operatorname{Pr}\left[t_{d}<t_{c, i}<t_{f, i}\right]}{\operatorname{Pr}\left[t_{c, i}<t_{f, i}\right]} \\
& =\frac{\int_{t_{d}=0}^{\infty} \int_{t_{c, i}=t_{d}}^{\infty} \int_{t_{f, i}=t_{c, i}}^{\infty} f_{f}\left(t_{f, i}\right) \lambda e^{-\lambda t_{c, i}} \theta e^{-\theta t_{d}} d t_{f, i} d t_{c, i} d t_{d}}{1-f_{f}^{*}(\lambda)} \\
& =\frac{\left(\frac{\theta}{\lambda+\theta}\right)+\left(\frac{\lambda}{\lambda+\theta}\right) f_{f}^{*}(\lambda+\theta)-f_{f}^{*}(\lambda)}{1-f_{f}^{*}(\lambda)} .
\end{aligned}
$$

Let $N_{I, r}\left(t_{d}\right)$ and $N_{I I, r}\left(t_{d}\right)$ denote $N_{r}\left(t_{d}\right)$ conditioning on Case I and Case II, respectively. Then $\operatorname{Pr}\left[N_{r}\left(t_{d}\right)=k\right]$ is expressed as

$$
\begin{aligned}
\operatorname{Pr}\left[N_{r}\left(t_{d}\right)=k\right] & =\operatorname{Pr}\left[N_{I, r}\left(t_{d}\right)=k\right] \operatorname{Pr}[\text { Case I }]+\operatorname{Pr}\left[N_{I I, r}\left(t_{d}\right)=k\right] \operatorname{Pr}[\text { Case II }] \\
& =\frac{\eta_{f} \operatorname{Pr}\left[N_{I, r}\left(t_{d}\right)=k\right]+\eta_{m} \operatorname{Pr}\left[N_{I I, r}\left(t_{d}\right)=k\right]}{\eta_{m}+\eta_{f}} .
\end{aligned}
$$

$\operatorname{Pr}\left[N_{I, r}\left(t_{d}\right)=k\right]$ and $\operatorname{Pr}\left[N_{I I, r}\left(t_{d}\right)=k\right]$ are derived as follows:

The derivation of $\operatorname{Pr}\left[N_{I, r}\left(t_{d}\right)=k\right]$ : Similar to the derivation for $\operatorname{Pr}\left[N_{I, c}=k\right]$, we consider Case Ia (i.e., the next call request arrives when the MS is in $L_{f}$ ) and Case $\mathrm{Ib}$ (i.e., the next call request arrives when the MS is in $\left.L_{m}\right)$ to derive $\operatorname{Pr}\left[N_{I, r}\left(t_{d}\right)=k\right]$.

Case Ia: The previous call request arrives during $t_{m, 0}$, and the next call request arrives during $t_{f, j+1}$. See Figure 2 (a). In this case, the MS has $N_{I, c}=2 j+1$ crossings. The MS visits the macrocell for $j$ times and the overlapped femtocell for $j+1$ times (i.e., $\left.t_{f, 1}, t_{m, 1}, t_{f, 2}, t_{m, 2}, \ldots, t_{f, j}, t_{m, j}, t_{f, j+1}\right)$. For $1 \leq i \leq j$, if the MS executes a registration during $t_{f, i}$ (i.e., $t_{d}<t_{f, i}$ ), then after $t_{f, i}$ (i.e., at the beginning of $t_{m, i}$ ), a registration 
will be executed. Otherwise (i.e., the MS does not execute a registration during $t_{f, i}$; i.e., $\left.t_{d}>t_{f, i}\right)$, after $t_{f, i}$ (i.e., at the beginning of $t_{m, i}$ ), no registration will be executed. Among $N_{I, c}=2 j+1$ crossings, for $N_{I, r}\left(t_{d}\right)=k$, if $k$ is an odd number, a registration is executed during $t_{f, j+1}$. Otherwise (i.e., $k$ is an even number), a registration is not executed during $t_{f, j+1}$. Then we have

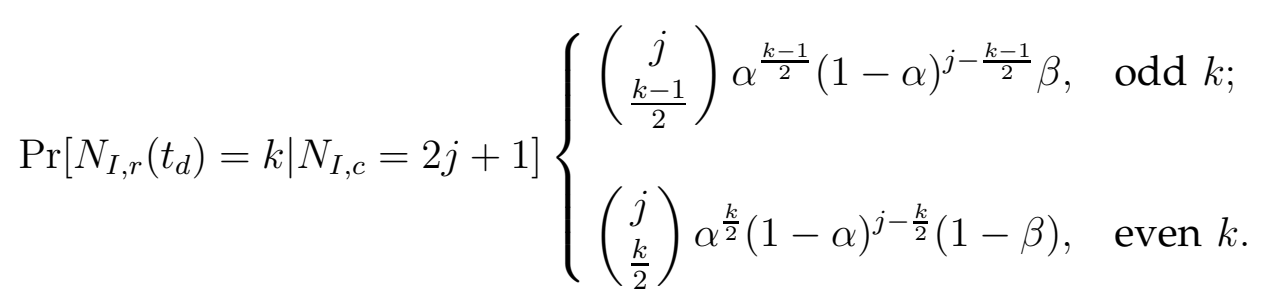

Case Ib: The previous call request arrives during $t_{m, 0}$, and the next call request arrives during $t_{m, j}$ where $j \geq 1$. See Figure $2(\mathrm{~b})$. In this case, the MS has $N_{I, c}=2 j$ crossings. The MS visits the overlapped femtocell for $j$ times and the macrocell for $j$ times (i.e., $\left.t_{f, 1}, t_{m, 1}, t_{f, 2}, t_{m, 2}, \ldots, t_{f, j}, t_{m, j}\right)$. Among $N_{I, c}=2 j$ crossings, for $N_{I, r}\left(t_{d}\right)=k, k$ must be an even number. Then, similar to the derivation of (21), we have

$$
\operatorname{Pr}\left[N_{I, r}\left(t_{d}\right)=k \mid N_{I, c}=2 j\right]=\left(\begin{array}{c}
j \\
\frac{k}{2}
\end{array}\right) \alpha^{\frac{k}{2}}(1-\alpha)^{j-\frac{k}{2}} .
$$

From (21) and (22), we have

$$
\operatorname{Pr}\left[N_{I, r}\left(t_{d}\right)=k\right]= \begin{cases}\sum_{j=\frac{k-1}{2}}^{\infty} \operatorname{Pr}\left[N_{I, r}\left(t_{d}\right)=k \mid N_{I, c}=2 j+1\right] \operatorname{Pr}\left[N_{I, c}=2 j+1\right] ; & \text { odd } k, \\ \sum_{j=\frac{k}{2}}^{\infty} \operatorname{Pr}\left[N_{I, r}\left(t_{d}\right)=k \mid N_{I, c}=2 j+1\right] \operatorname{Pr}\left[N_{I, c}=2 j+1\right] & \\ +\sum_{j=\frac{k}{2}}^{\infty} \operatorname{Pr}\left[N_{I, r}\left(t_{d}\right)=k \mid N_{I, c}=2 j\right] \operatorname{Pr}\left[N_{I, c}=2 j\right], & \text { even } k .\end{cases}
$$

Applying (12), (13), (21) and (22) into (23), we obtain

$$
\operatorname{Pr}\left[N_{I, r}\left(t_{d}\right)=k\right]=\left\{\begin{array}{l}
\frac{\eta_{m} \beta\left[1-f_{m}^{*}(\lambda)\right]\left[1-f_{f}^{*}(\lambda)\right]\left[\alpha f_{m}^{*}(\lambda) f_{f}^{*}(\lambda)\right]^{\frac{k-1}{2}}}{\lambda\left[1-(1-\alpha) f_{m}^{*}(\lambda) f_{f}^{*}(\lambda)\right]^{\frac{k+1}{2}}}, \\
\frac{\eta_{m}\left[1-f_{m}^{*}(\lambda)\right]\left[1-(1-\beta) f_{m}^{*}(\lambda) f_{f}^{*}(\lambda)-\beta f_{m}^{*}(\lambda)\right]\left[\alpha f_{m}^{*}(\lambda) f_{f}^{*}(\lambda)\right]^{\frac{k}{2}}}{\lambda f_{m}^{*}(\lambda)\left[1-(1-\alpha) f_{m}^{*}(\lambda) f_{f}^{*}(\lambda)\right]^{\frac{k}{2}+1}},
\end{array}\right.
$$
odd $k$; even $k$. 
The derivation of $\operatorname{Pr}\left[N_{I I, r}\left(t_{d}\right)=k\right]$ : We consider Case IIa (i.e., the next call request arrives when the MS is in $L_{f}$ ) and Case IIb (i.e., the next call request arrives when the MS is in $\left.L_{m}\right)$ to derive $\operatorname{Pr}\left[N_{I I, r}\left(t_{d}\right)=k\right]$.

Case IIa: The previous call request arrives during $t_{f, 0}$, and the next call request arrives during $t_{f, j}$ where $j \geq 1$. See Figure 2 (c). In this case, the MS has $N_{I I, c}=2 j$ crossings. The MS visits the overlapped femtocell for $j$ times and the macrocell for $j$ times (i.e., $\left.t_{m, 1}, t_{f, 1}, t_{m, 2}, t_{f, 2}, \ldots, t_{m, j}, t_{f, j}\right)$. At the beginning of $t_{m, 1}$ (after $\left.t_{f, 0}\right)$, the MS executes a registration if the MS has executed a registration during $t_{f, 0}$. For $1 \leq i<j$, if the MS executes a registration during $t_{f, i}$ (i.e., $t_{d}<t_{f, i}$ ), then after $t_{f, i}$ (i.e., at the beginning of $\left.t_{m, i+1}\right)$, a registration is executed. Otherwise (i.e., the MS does not execute a registration during $t_{f, i}$; i.e., $\left.t_{d}>t_{f, i}\right)$, then after $t_{f, i}$, at the beginning of $t_{m, i+1}$, no registration is executed.

Among $N_{I I, c}=2 j$ crossings, for $N_{I I, r}\left(t_{d}\right)=k$, if $k$ is an odd number, a registration is executed during $t_{f, 0}$ and no registration is executed during $t_{f, j}$, or no registration is executed during $t_{f, 0}$ and a registration is executed during $t_{f, j}$. Otherwise (i.e., $k$ is an even number), the registrations are executed during $t_{f, 0}$ and $t_{f, j}$, or no registration is executed neither during $t_{f, 0}$ nor during $t_{f, j}$. Then we have

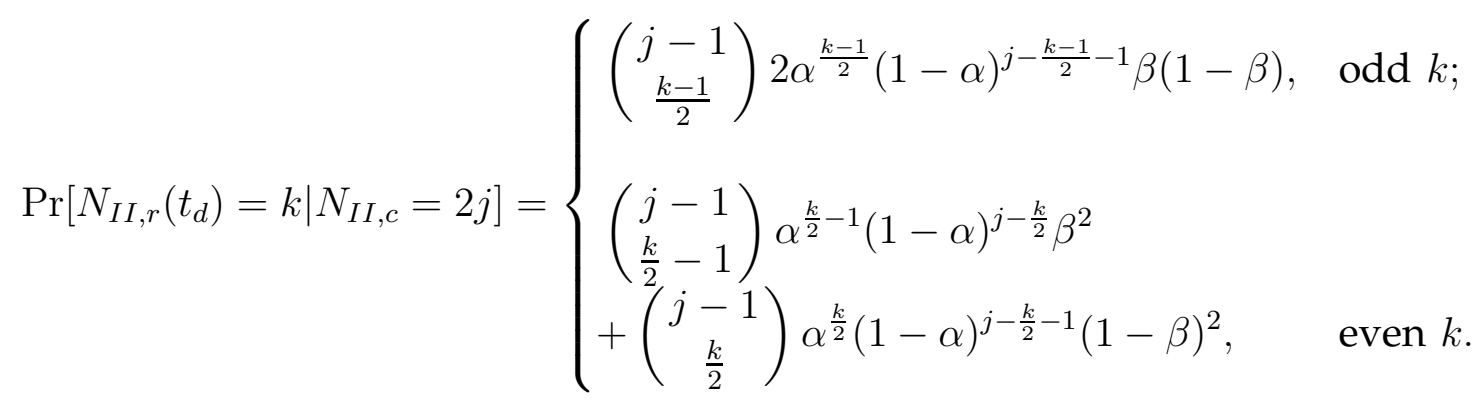

Case IIb: The previous call request arrives during $t_{f, 0}$, and the next call request arrives during $t_{m, j+1}$ where $j \geq 0$. See Figure $2(\mathrm{~d})$. In this case, the MS has $N_{I I, c}=2 j+1$ crossings where the MS visits the overlapped femtocell for $j$ times and the macrocell for $j+1$ times (i.e., $t_{m, 1}, t_{f, 1}, t_{m, 2}, t_{f, 2}, \ldots, t_{m, j}, t_{f, j}, t_{m, j+1}$ ). Among $N_{I I, c}=2 j+1$ crossings, for $N_{I I, r}\left(t_{d}\right)=k$, if $k$ is an odd number, a registration is executed during $t_{f, 0}$. Otherwise (i.e., $k$ is an even number), no registration is executed during $t_{f, 0}$. Then 
we have

$$
\operatorname{Pr}\left[N_{I I, r}\left(t_{d}\right)=k \mid N_{I I, c}=2 j+1\right]= \begin{cases}\left(\begin{array}{c}
j \\
\frac{k-1}{2}
\end{array}\right) \alpha^{\frac{k-1}{2}}(1-\alpha)^{j-\frac{k-1}{2}} \beta, & \text { odd } k ; \\
\left(\begin{array}{c}
j \\
\frac{k}{2}
\end{array}\right) \alpha^{\frac{k}{2}}(1-\alpha)^{j-\frac{k}{2}}(1-\beta), & \text { even } k .\end{cases}
$$

From (25) and (26), we rewrite

$$
\operatorname{Pr}\left[N_{I I, r}\left(t_{d}\right)=k\right]= \begin{cases} & \sum_{j=\frac{k+1}{2}}^{\infty} \operatorname{Pr}\left[N_{I I, r}\left(t_{d}\right)=k \mid N_{I I, c}=2 j\right] \operatorname{Pr}\left[N_{I I, c}=2 j\right] \\ + & \sum_{j=\frac{k-1}{2}}^{\infty} \operatorname{Pr}\left[N_{I I, r}\left(t_{d}\right)=k \mid N_{I I, c}=2 j+1\right] \operatorname{Pr}\left[N_{I I, c}=2 j+1\right], \quad \text { odd } k ; \\ \sum_{j=\frac{k}{2}}^{\infty} \operatorname{Pr}\left[N_{I I, r}\left(t_{d}\right)=k \mid N_{I I, c}=2 j\right] \operatorname{Pr}\left[N_{I I, c}=2 j\right] & \text { even } k . \\ +\sum_{j=\frac{k}{2}}^{\infty} \operatorname{Pr}\left[N_{I I, r}\left(t_{d}\right)=k \mid N_{I I, c}=2 j+1\right] \operatorname{Pr}\left[N_{I I, c}=2 j+1\right], & \end{cases}
$$

Apply (14), (15), (25) and (26) into (27) to yield

$$
\operatorname{Pr}\left[N_{I I, r}\left(t_{d}\right)=k\right]=\left\{\begin{array}{l}
\left(\frac{\eta_{f} \beta\left[1-f_{f}^{*}(\lambda)\right]\left[\alpha f_{m}^{*}(\lambda) f_{f}^{*}(\lambda)\right]^{\frac{k-1}{2}}}{\lambda\left[1-(1-\alpha) f_{m}^{*}(\lambda) f_{f}^{*}(\lambda)\right]^{\frac{k+1}{2}}}\right) \\
{\left[1+(1-2 \beta) f_{m}^{*}(\lambda)-2(1-\beta) f_{m}^{*}(\lambda) f_{f}^{*}(\lambda)\right],} \\
\left(\begin{array}{ll}
\eta_{f}\left[1-f_{f}^{*}(\lambda)\right]\left[\alpha f_{m}^{*}(\lambda) f_{f}^{*}(\lambda)\right]^{\frac{k}{2}} \\
\lambda \alpha f_{f}^{*}(\lambda)\left[1-(1-\alpha) f_{m}^{*}(\lambda) f_{f}^{*}(\lambda)\right]^{\frac{k}{2}+1}
\end{array}\right) \\
\left\{\beta\left[1-f_{f}^{*}(\lambda)\right]\left[(\alpha+\beta-2 \alpha \beta) f_{m}^{*}(\lambda) f_{f}^{*}(\lambda)-\beta\right]\right. \\
\left.-\alpha(1-\beta) f_{f}^{*}(\lambda)\left[1-f_{m}^{*}(\lambda) f_{f}^{*}(\lambda)\right]\right\},
\end{array}\right.
$$

The probability $\operatorname{Pr}\left[N_{r}\left(t_{d}\right)=k\right]$ can be obtained by applying (24) and (28) into (20). Then we have

$$
E\left[N_{r}\left(t_{d}\right)\right]=\sum_{k=1}^{\infty} k \operatorname{Pr}\left[N_{r}\left(t_{d}\right)=k\right]=\frac{2 \eta_{m} \eta_{f}\left[\beta+(\alpha-\beta) f_{f}^{*}(\lambda)\right]}{\lambda\left(\eta_{m}+\eta_{f}\right)(\lambda+\theta)}
$$

Applying (18) and (19) into (29), we have

$$
E\left[N_{r}\left(t_{d}\right)\right]=\frac{2 \eta_{m} \eta_{f} \theta\left[1-f_{f}^{*}(\lambda+\theta)\right]}{\lambda\left(\eta_{m}+\eta_{f}\right)(\lambda+\theta)}
$$




\subsection{Derivation of Potential Offload Traffic Ratio $\rho\left(t_{d}\right)$}

In the 3GPP algorithms, the LAI stored in the location database is $L_{f}$ as long as the MS is in the overlapped femtocell, and the same applies for $L_{m}$ as long as the MS is in the macrocell. When a call request arrives during $t_{f}$ and $t_{m}$, by querying the LAI information in the location database, the call request is processed by the overlapped femtocell and macrocell, respectively. Since the MS alters between $t_{m}$ and $t_{f}$, from the alternating renewal process [20], the probability $p(0)$ that a call arrives during $t_{f}$ is

$$
p(0)=\frac{E\left[t_{f}\right]}{E\left[t_{m}\right]+E\left[t_{f}\right]}=\frac{\eta_{m}}{\eta_{m}+\eta_{f}} .
$$

As shown in Figure 2, the MS shifts between staying in the macrocell and the overlapped femtocell for $t_{m}$ and $t_{f}$. Suppose that the MS moves from the macrocell to the overlapped femtocell at $t$, and leaves the femtocell at $t+t_{f}$. In the DR algorithm, at $t$, the registration is suspended until the delay timer with length $t_{d}$ expires. During the period $\left[t, t+\min \left(t_{d}, t_{f}\right)\right]$, the LAI stored in the database is $L_{m}$, and if a call request arrives, it is processed by the macrocell. During the period $\left[t+\min \left(t_{d}, t_{f}\right), t+t_{f}\right]$, the LAI stored in the database is $L_{f}$, and if a call request request arrives, it is processed by the femtocell. Let $t_{m}^{\prime}=t_{m}+\min \left(t_{d}, t_{f}\right)$, and $t_{f}^{\prime}=t_{f}-\min \left(t_{d}, t_{f}\right)$, and we have $E\left[t_{m}^{\prime}\right]=E\left[t_{m}\right]+E\left[\min \left(t_{d}, t_{f}\right)\right]$ and $E\left[t_{f}^{\prime}\right]=E\left[t_{f}\right]-$ $E\left[\min \left(t_{d}, t_{f}\right)\right]$. In other words, the LAI is alternatively changed between $t_{m}^{\prime}$ and $t_{f}^{\prime}$. During $t_{f}^{\prime}$, all call request arrivals are processed by the femtocell. Therefore, $p\left(t_{d}\right)$ is the probability that a call arrives during $t_{f}^{\prime}$. According to the alternating renewal process, we have

$$
\begin{aligned}
p\left(t_{d}\right) & =\frac{E\left[t_{f}^{\prime}\right]}{E\left[t_{m}^{\prime}\right]+E\left[t_{f}^{\prime}\right]}=\frac{E\left[t_{f}\right]-E\left[\min \left(t_{d}, t_{f}\right)\right]}{\left(E\left[t_{m}\right]+E\left[\min \left(t_{d}, t_{f}\right)\right]\right)+\left(E\left[t_{f}\right]-E\left[\min \left(t_{d}, t_{f}\right)\right]\right)} \\
& =\frac{E\left[t_{f}\right]-E\left[\min \left(t_{d}, t_{f}\right)\right]}{E\left[t_{m}\right]+E\left[t_{f}\right]} .
\end{aligned}
$$

where

$$
\begin{aligned}
E\left[\min \left(t_{d}, t_{f}\right)\right] & =\int_{t_{f}=0}^{\infty} \int_{t_{d}=0}^{t_{f}} t_{d} f_{f}\left(t_{f}\right) \theta e^{-\theta t_{d}} d t_{d} d t_{f}+\int_{t_{f}=0}^{\infty} \int_{t_{d}=t_{f}}^{\infty} t_{f} f_{f}\left(t_{f}\right) \theta e^{-\theta t_{d}} d t_{d} d t_{f} \\
& =\frac{1-f_{f}^{*}(\theta)}{\theta}
\end{aligned}
$$


Then (32) is rewritten as

$$
p\left(t_{d}\right)=\frac{\eta_{m}\left(\theta-\eta_{f}+\eta_{f} f_{f}^{*}(\theta)\right)}{\theta\left(\eta_{m}+\eta_{f}\right)} .
$$

Then we apply (31) and (33) into (2) to yield

$$
\rho\left(t_{d}\right)=\frac{\theta-\eta_{f}+\eta_{f} f_{f}^{*}(\theta)}{\theta} .
$$

\section{Performance Evaluation}

In this section, we first analyze the accuracy of the analytical models proposed in this paper through the simulation experiments, and then study the $r\left(t_{d}\right)$ and $\rho\left(t_{d}\right)$ performances of the DR algorithm.

As shown in (3) and (34), $r\left(t_{d}\right)$ and $\rho\left(t_{d}\right)$ are obtained if the closed-form expressions of $f_{m}^{*}(s)$ and $f_{f}^{*}(s)$ exist. In this study, we apply the Gamma distribution for the residence times $t_{m}$ and $t_{f}$. The Gamma distribution is selected because it can approximate many types of distributions, it has a closed-form expression for its Laplace transform, and it has been widely used in many previous works (e.g., [9-11]) to reflect the MS mobility.

Suppose that the Gamma density functions $f_{m}(t)$ and $f_{f}(t)$ are with the means $1 / \eta_{m}$ and $1 / \eta_{f}$, the shape parameters $\gamma_{m}$ and $\gamma_{f}$, the variances $v_{m}=1 /\left(\gamma_{m} \eta_{m}^{2}\right)$ and $v_{f}=1 /\left(\gamma_{f} \eta_{f}^{2}\right)$, and the Laplace transforms

$$
f_{m}^{*}(s)=\left(\frac{\gamma_{m} \eta_{m}}{\gamma_{m} \eta_{m}+s}\right)^{\gamma_{m}} \text { and } f_{f}^{*}(s)=\left(\frac{\gamma_{f} \eta_{f}}{\gamma_{f} \eta_{f}+s}\right)^{\gamma_{f}} .
$$

Then we have

$$
r\left(t_{d}\right)=\frac{\theta-\theta\left(\frac{\gamma_{f} \eta_{f}}{\gamma_{f} \eta_{f}+\lambda+\theta}\right)^{\gamma_{f}}}{\lambda+\theta},
$$

and

$$
\rho\left(t_{d}\right)=\frac{\theta-\eta_{f}+\eta_{f}\left(\frac{\gamma_{f} \eta_{f}}{\gamma_{f} \eta_{f}+\theta}\right)^{\gamma_{f}}}{\theta} .
$$

We develop the simulation model for the DR algorithm based on the discrete eventdriven approach, one widely used in MCN studies (e.g., [17], [21]). The simulation model is similar to that in [17], so the details are not presented in this paper. In our study, the input 
TABLE 2

Validation of the simulation and analysis results

\begin{tabular}{|c|c|c|c|c|c|c|}
\hline \multicolumn{6}{|c|}{$E\left[t_{c}\right]=1 / \lambda=50$ minutes, $\eta_{f}=200 \lambda, v_{f}=1000 / \eta_{f}^{2}, \eta_{m}=50 \lambda, v_{m}=10 / \eta_{m}^{2}$} \\
\hline$E\left[t_{d}\right]$ (unit: $\left.1 / \lambda\right)$ & $1 / 10$ & $1 / 20$ & $1 / 30$ & $1 / 40$ & $1 / 50$ \\
\hline \multirow{3}{*}{$\alpha$} & Analytic & 0.0022311 & 0.00286756 & 0.00325279 & 0.00352987 & 0.00374638 \\
\cline { 2 - 7 } & Simulation & 0.00223434 & 0.00287484 & 0.0032586 & 0.00353166 & 0.00375318 \\
\cline { 2 - 7 } & Error (\%) & 0.145318 & 0.253703 & 0.178593 & 0.0506395 & 0.18136 \\
\hline \multirow{4}{*}{$\beta$} & Analytic & 0.795992 & 0.876239 & 0.909232 & 0.927603 & 0.939431 \\
\cline { 2 - 7 } & Simulation & 0.796412 & 0.875418 & 0.909573 & 0.927354 & 0.939321 \\
\cline { 2 - 7 } & Error (\%) & 0.0527235 & 0.0936923 & 0.0374611 & 0.0268176 & 0.0117352 \\
\hline \multirow{3}{*}{$\rho\left(t_{d}\right)$} & Analytic & 0.00365206 & 0.00443103 & 0.00487464 & 0.00518411 & 0.0054214 \\
\cline { 2 - 7 } & Simulation & 0.0036542 & 0.00441135 & 0.00488304 & 0.00520538 & 0.00543302 \\
\cline { 2 - 7 } & Error (\%) & 0.0587348 & 0.444215 & 0.172404 & 0.410383 & 0.214335 \\
\hline \multirow{3}{*}{$\rho\left(t_{d}\right)$} & Analytic & 0.921518 & 0.953955 & 0.966635 & 0.973554 & 0.977959 \\
\cline { 2 - 7 } & Simulation & 0.921672 & 0.95301 & 0.966418 & 0.97411 & 0.977663 \\
\cline { 2 - 7 } & Error (\%) & 0.0167304 & 0.0990423 & 0.0225196 & 0.0571416 & 0.0303185 \\
\hline
\end{tabular}

parameters $\eta_{m}, \eta_{f}$ and $\theta$ are normalized by $\lambda$. For example, if we set the expected inter-call arrival time $E\left[t_{c}\right]=1 / \lambda=50$ minutes [14], $\theta=10 \lambda$ means that the expected delay period $E\left[t_{d}\right]=1 / \theta=5$ minutes. In other words, our study results are dependent on the call arrival rate $\lambda$. For the DR algorithm to be effective, $\lambda$ must be estimated very accurately. Accurate $\lambda$ measurements are available in existing core network nodes (i.e., mobile switching centers or GPRS support nodes).

The analytical and simulation results are validated against each other. As shown in Table 2, the errors between the analytical and simulation results fall within $1 \%$, demonstrating consistent findings from both our analytical models and simulation experiments.

In the following, we investigate the effects of input parameters on $r\left(t_{d}\right)$ and $\rho\left(t_{d}\right)$ for the DR algorithm. Section 4.1 studies the effects of MS mobility. Section 4.2 studies the effects of $t_{d}$ with fixed and exponential setups.

\subsection{Effects of MS Mobility}

This section studies the effects of MS mobility behaviors on the $r\left(t_{d}\right)$ and $\rho\left(t_{d}\right)$ performances. From (3) and (34), it is clear that $r\left(t_{d}\right)$ and $\rho\left(t_{d}\right)$ are independent from the distribution for $t_{m}$, indicating that the MS mobility behavior in non-overlapped areas of the macrocell does not affect the $r\left(t_{d}\right)$ and $\rho\left(t_{d}\right)$ performances. Therefore, in the following, we only study the effects of MS mobility behavior in the overlapped femtocell. 


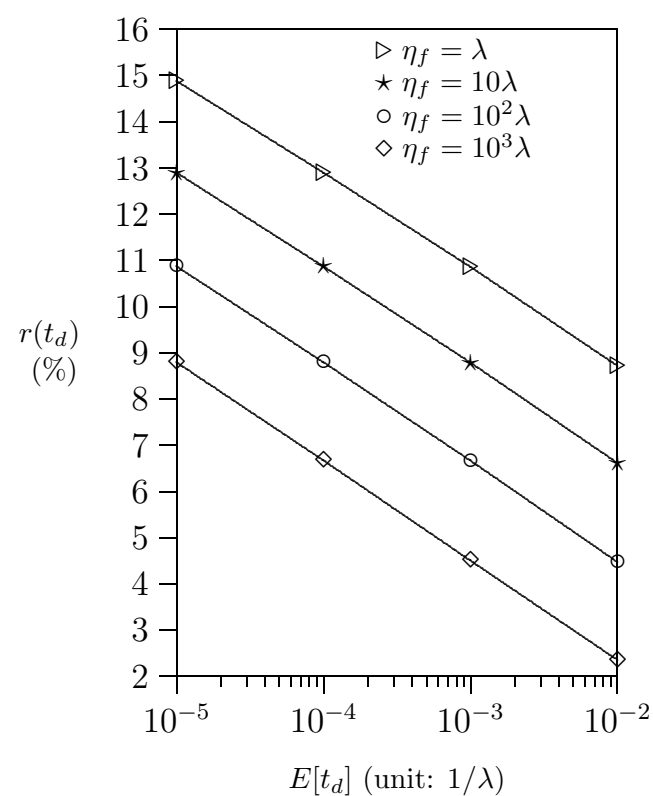

(a) Performance of Signaling Overhead Ratio

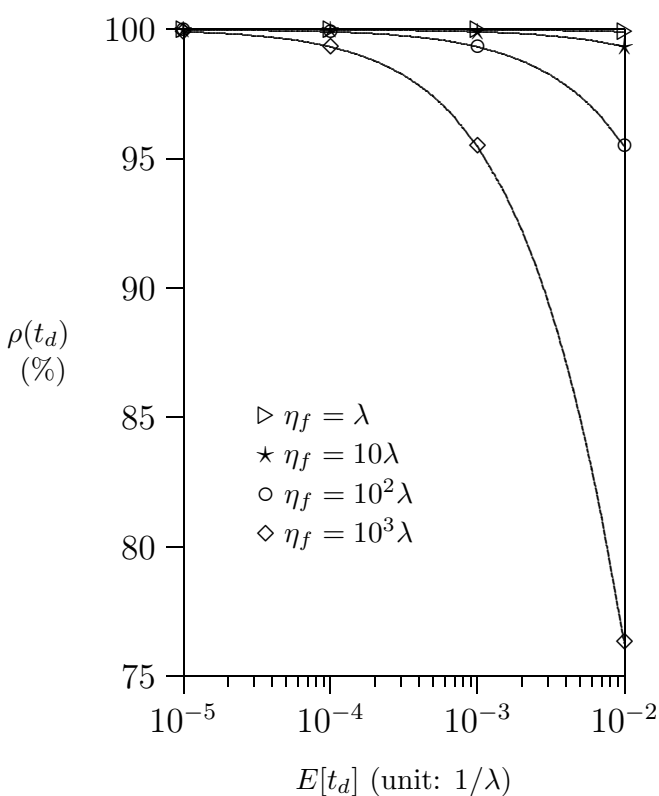

(b) Performance of Potential Offload Traffic Ratio

Fig. 3. The effects of $\eta_{f}$ on $r\left(t_{d}\right)$ and $\rho\left(t_{d}\right)\left(\eta_{m}=25 \lambda, v_{m}=1 / \eta_{m}^{2}, v_{f}=100 / \eta_{f}^{2}\right)$

Effects of Mean of Femtocell Residence Time $1 / \eta_{f}$ : In Figure 3, we study the effects of the femtocell residence time, where $E\left[t_{d}\right]$ is set from $10^{-5} / \lambda$ (i.e., 0.03 seconds) to $10^{-2} / \lambda$ (i.e., 30 seconds), $\eta_{m}=25 \lambda$ (i.e., $E\left[t_{m}\right]=2$ minutes), and $v_{m}=1 / \eta_{m}^{2}$. We set $v_{f}=100 / \eta_{f}^{2}$ to simulate the real MS mobility behavior that the MS either stays in the femtocell for a long period or just passes by the femtocell.

As shown in Figure 3 (a), $r\left(t_{d}\right)$ decreases as $\eta_{f}$ increases. A larger $\eta_{f}$ implies that the MS stays in the overlapped femtocell for a shorter period. This is when a transient phenomenon might likely occur, during which more registration traffic can be avoided in the DR algorithm. Figure 3 (a) also indicates that the longer we set the delay timer, the more registration avoided, i.e., $r\left(t_{d}\right)$ decreases as $E\left[t_{d}\right]$ increases. In Figure 3 (a), we observe that the DR algorithm reduces at least $85 \%$ of registration signaling overhead. On the other hand, in Figure $3(\mathrm{~b}), \rho\left(t_{d}\right)$ decreases as $\eta_{f}$ increases, i.e., with the delay timer, the transient phenomenon reduces traffic offloading capability of the femtocell. We observe that the DR algorithm causes at most $24 \%$ of the degradation of the traffic offloading capability (i.e., $\rho\left(t_{d}\right) \approx 76 \%$ when $\eta_{f}=10^{3} \lambda$ and $E\left[t_{d}\right]=10^{-2} / \lambda$ ). To summarize, 
the DR algorithm significantly reduces the registration signaling overhead. Meanwhile, the DR algorithm sustains good performance for the traffic offloading capability of the femtocell.

In the following, we discuss how to set up the delay timer adapting to different MS mobility behaviors to achieve better $r\left(t_{d}\right)$ performance while minimizing the loss in traffic offload capability. Observe the " $\diamond$ " curves in Figure 3 (a) and (b), where $\eta_{f}=10^{3} \lambda$ (i.e., $E\left[t_{f}\right]=3$ seconds). In this mobility scenario, the MS stays in the overlapped femtocell for transient periods. Figure 3 (a) indicates how the $r\left(t_{d}\right)$ values drop linearly (i.e., better $r\left(t_{d}\right)$ performance is obtained; from $9 \%$ to $2.3 \%$ ) as $E\left[t_{d}\right]$ increases from $10^{-5} / \lambda$ to $10^{-2} / \lambda$. However, Figure $3(\mathrm{~b})$ indicates that when $E\left[t_{d}\right] \leq 10^{-3} / \lambda$, the $\rho\left(t_{d}\right)$ performance decreases slightly (from $100 \%$ to $95 \%$ ) as $E\left[t_{d}\right]$ increases, but when $E\left[t_{d}\right]>$ $10^{-3} / \lambda$, the $\rho\left(t_{d}\right)$ performance drops very quickly (from $95 \%$ to $76 \%$ ). To summarize, we prefer to set $E\left[t_{d}\right] \leq 10^{-3} / \lambda$ (i.e., 3 seconds).

For other mobility scenarios, $\eta_{f}=\lambda, \eta_{f}=10 \lambda$, and $\eta_{f}=100 \lambda$ (see " $\triangleright$ ", " $\star$ ", and "०" curves), we prefer to set $E\left[t_{d}\right]=10^{-2} / \lambda$ (i.e., 30 seconds) because when $E\left[t_{d}\right]=10^{-2} / \lambda$, we achieve the best $r\left(t_{d}\right)$ performance with loss of traffic offloading capability no larger than $5 \%$ (i.e., $\rho\left(t_{d}\right)=95 \%$ ).

Effects of Variance of Femtocell Residence Time $v_{f}$ : In Figure 4, we study the effects of the variance $v_{f}$ of the femtocell residence time, where $E\left[t_{d}\right]$ is set from $10^{-5} / \lambda$ (i.e., 0.03 seconds) to $10^{-2} / \lambda$ (i.e., 30 seconds), $\eta_{m}=25 \lambda, \eta_{f}=100 \lambda$, and $v_{m}=1 / \eta_{m}^{2}$. As $v_{f}$ increases, it is more likely to observe an MS with short and long residence time in an overlapped femtocell, so the MS mobility behavior in the femtocell is more "dynamic". For short $t_{f}$ periods, it is more likely that an MS moves out of an overlapped femtocell before the delay timer $t_{d}$ expires. The MS has less chance to execute the registration in the overlapped femtocell, reducing more signaling overhead caused by registration. Therefore, we observe $r\left(t_{d}\right)$ decreases as $v_{f}$ increases in Figure 4 (a).

On the other hand, for longer $t_{f}$ periods, the MS is more likely to have call requests 


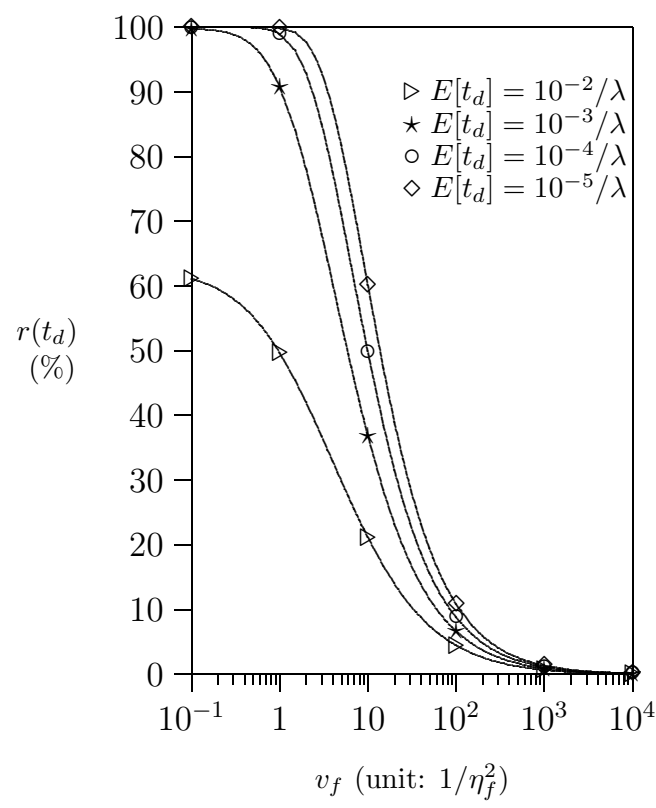

(a) Performance of Signaling Overhead Ratio

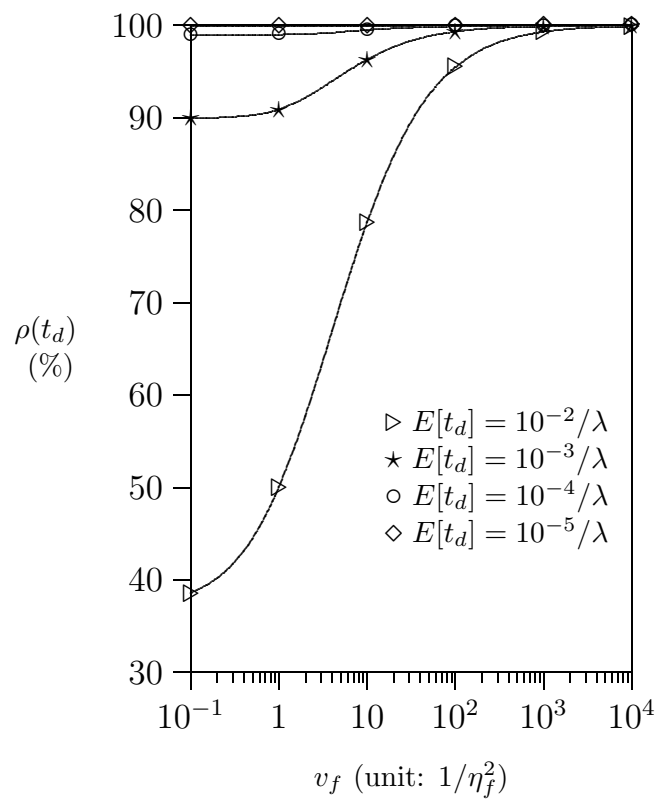

(b) Performance of Potential Offload Traffic Ratio

Fig. 4. The effects of $v_{f}$ on $r\left(t_{d}\right)$ and $\rho\left(t_{d}\right)\left(\eta_{m}=25 \lambda, \eta_{f}=100 \lambda, v_{m}=1 / \eta_{m}^{2}\right)$

through the long-residence femtocell. More requests are potentially processed by the femtocell. Therefore, larger $\rho\left(t_{d}\right)$ is observed as $v_{f}$ increases in Figure 4 (b).

To summarize, when the MS mobility is more dynamic, the DR algorithm can work more effectively (i.e., both $r\left(t_{d}\right)$ and $\rho\left(t_{d}\right)$ have better performance when $v_{f}$ is larger).

In addition to the Gamma distribution, in this study, we also considered the Weibull distribution for MS mobility behaviors, which has also been widely used to approximate real MS mobility patterns in many MCN studies (e.g., [22], [23]). Note that the Weibull distribution does not have a closed-form expression for its Laplace transform [24]. Therefore, the closed-form expressions of $r\left(t_{d}\right)$ and $\rho\left(t_{d}\right)$ for the Weibull distribution do not exist in our analytical models. Instead, we run simulation experiments to study effects of MS mobility on $r\left(t_{d}\right)$ and $\rho\left(t_{d}\right)$ for the Weibull distributed residence times. We observe similar performance trends for both the Weibull and Gamma distributions, and thus we do not include the performance evaluation for the Weibull distributed residence times. 


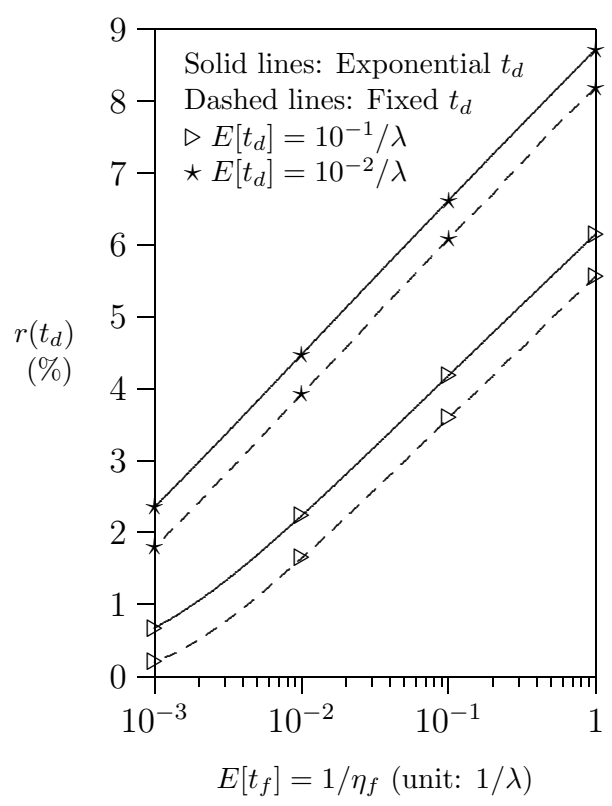

(a) Performance of Signaling Overhead Ratio (b)

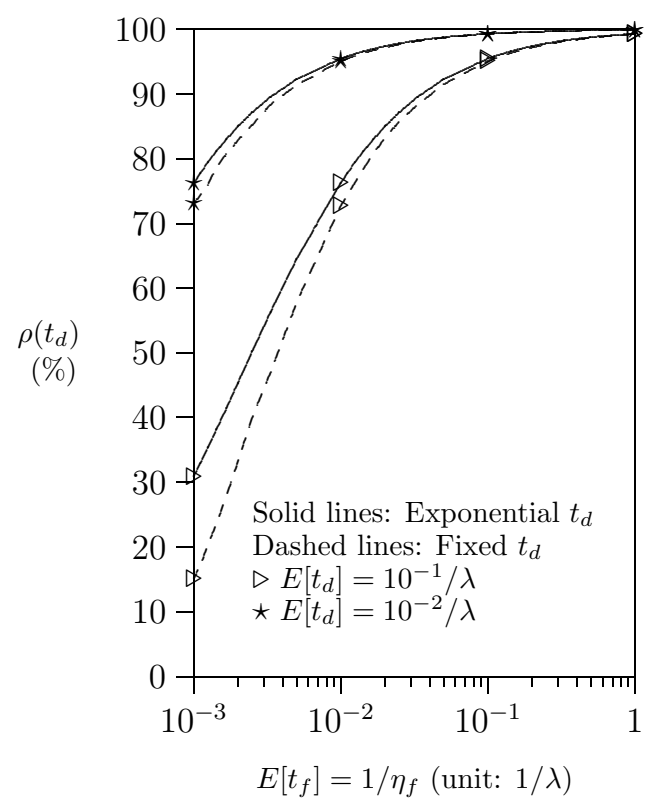

(b) Performance of Potential Offload Traffic Ratio

Fig. 5. The effects of fixed and exponential $t_{d}$ on $r\left(t_{d}\right)$ and $\rho\left(t_{d}\right)\left(\eta_{m}=25 \lambda, v_{m}=1 / \eta_{m}^{2}\right.$, $\left.v_{f}=100 / \eta_{f}^{2}\right)$.

\subsection{Effects of Fixed and Exponential $t_{d}$}

In Figure 5, based on the simulation experiments, we study $r\left(t_{d}\right)$ and $\rho\left(t_{d}\right)$ against $E\left[t_{f}\right]$ for fixed and exponential $t_{d}$, where $\eta_{m}=25 \lambda, v_{m}=1 / \eta_{m}^{2}, v_{f}=100 / \eta_{f}^{2}$, and $t_{f}$ is Gamma distributed. We observe that the performance trends of $r\left(t_{d}\right)$ and $\rho\left(t_{d}\right)$ for fixed $t_{d}$ are similar to those for exponential $t_{d}$.

In Figure 5 (a), when $E\left[t_{d}\right]=10^{-1} / \lambda$ and $E\left[t_{d}\right]=10^{-2} / \lambda, r\left(t_{d}\right)$ for fixed $t_{d}$ is about $0.5 \%$ lower than that for exponential $t_{d}$. As $E\left[t_{f}\right]$ increases from $10^{-3} / \lambda$ to $1 / \lambda$, this difference remains the same.

On the other hand, in Figure $5(\mathrm{~b})$, as $E\left[t_{f}\right]$ increases from $10^{-3} / \lambda$ to $1 / \lambda$, the difference between $\rho\left(t_{d}\right)$ of fixed $t_{d}$ and that of exponential $t_{d}$ diminishes from $2.5 \%$ to $0 \%$ for $E\left[t_{d}\right]=$ $10^{-2} / \lambda$, and from $15 \%$ to $0 \%$ for $E\left[t_{d}\right]=10^{-1} / \lambda$. This difference is larger when $E\left[t_{d}\right]$ is longer.

To summarize, the performance trends for $E\left[t_{d}\right]=10^{-1} / \lambda$ and $E\left[t_{d}\right]=10^{-2} / \lambda$ are very similar. To achieve better $r\left(t_{d}\right)$ and $\rho\left(t_{d}\right)$, we suggest to use exponential $t_{d}$ setup when $E\left[t_{f}\right]<10^{-1} / \lambda$ and use fixed $t_{d}$ setup when $E\left[t_{f}\right] \geq 10^{-1} / \lambda$. 


\section{Conclusions}

In this paper, we proposed a Delay Registration (DR) algorithm to reduce signaling overhead caused by frequent registrations, while noticing the slight decrease in traffic offloading capability of femtocells. To avoid registrations during the transient period, in the DR algorithm, we introduce a delay timer to postpone the registration until the timer expires. We conducted analytical models and simulation experiments to study the performance of the DR algorithm in terms of the signaling overhead ratio $r\left(t_{d}\right)$ and potential offload traffic ratio $\rho\left(t_{d}\right)$. The analytical model is general enough to accommodate various MS mobility behaviors. Our performance study can provide network operators with guidelines to configure the delay timer. Our study indicates that the DR algorithm can significantly reduce the signaling overhead with slight loss of traffic offloading capability of the femtocells. Moreover when the MS mobility is more dynamic, the DR algorithm can work more effectively, i.e., lower signaling overhead ratio and higher potential offload traffic ratio.

\section{ACKNOWLEDGEMENT}

The authors would like to express thanks to the four anonymous reviewers for their valuable comments. Their comments have significantly improved the quality of this paper.

\section{REFERENCES}

[1] 3GPP, “3rd Generation Partnership Project; Technical Specification Group Radio Access Network; UTRAN Architecture for 3G Home Node B (HNB); Stage 2 (Release 9)," Tech. Rep. 3G TS 25.467, 3GPP, September 2009.

[2] Femto Forum. Femtocells, http://www.femtoforum.org.

[3] Chandrasekhar, V., Andrews, J. G., and Gatherer, A., "Femtocell Networks: A Survey," IEEE Communications Magazine, vol. 46, pp. 59-67, September 2008.

[4] 3GPP, "3rd Generation Partnership Project; Technical Specification Group Services and Systems Aspects; Network architecture (Release 9)," Tech. Rep. 3G TS 23.002, 3GPP, November 2009.

[5] Broad Forum. Broadband, http://www.broadband-forum.org.

[6] 3GPP, "3rd Generation Partnership Project; Technical Specification Group Core Network and Terminals; Location Management Procedures (Release 8)," Tech. Rep. 3G TS 23.012, 3GPP, September 2009.

[7] Claussen, H., Ashraf, I., and Ho, L.T.W., "Dynamic Idle Mode Procedures for Femtocells," Bell Labs Technical Journal, vol. 15, pp. 95-116, September 2010.

[8] Lei, Y. and Zhang, Y., "Efficient Location Management Mechanism for Overlay LTE Macro and Femto Cells," Proceedings of IEEE ICCTA'09 Conference, pp. 420-424, December 2009.

[9] Akyildiz, I. F., Ho, J. S. M., and Lin, Y.-B., "Movement-based Location Update and Selective Paging for PCS Networks," IEEE/ACM Transactions on Networking, vol. 4, pp. 629-638, Augest 1996.

[10] Lin, Y.-B., "Reducing Location Update Cost in a PCS Network," IEEE/ACM Transations on Networking, vol. 5, pp. 25-33, February 1997.

[11] Lin, P. and Lin, Y.-B., "Implementation and Performance Evaluation for Mobility Management of a Wireless PBX Network," IEEE Journal on Selected Areas in Communications, vol. 19, pp. 1138-1146, June 2001.

[12] Xiao, Y. and Guizani, M., "Optimal Paging Load Balance with Total Delay Constraint in Macrocell-Microcell Hierarchical Cellular Networks," IEEE Transactions on Wireless Communications, vol. 5, pp. 2202-2209, August 2006. 
[13] Wu, X., Mukherjee, B., and Bhargava, B., "A Crossing-Tier Location Update/Paging Scheme in Hierarchical Cellular Networks," IEEE Transactions on Wireless Communications, vol. 5, pp. 839-848, April 2006.

[14] Park, K. I. and Lin, Y.-B., "Reducing Registration Traffic for Multi-tier Personal Communications Services," IEEE Transactions on Vehicular Technology, vol. 46, pp. 597-602, August 1997.

[15] Lin, Y.-B. and Chlamtac, I., Wireless and Mobile Network Architectures. John Wiley and Sons, 2001.

[16] Fang, Y., "Movement-based Mobility Management and Trade Off Analysis for Wireless Mobile Networks," IEEE Transactions on Computers, vol. 52, pp. 791-803, June 2003.

[17] Lin, P., Lin, Y.-B., and Jeng, J.-Y., "Improving GSM Call Completion by Call Re-Establishment," IEEE Journal on Selected Areas in Communications, vol. 17, pp. 1305-1317, July 1999.

[18] Lin, Y.-B. and Lin, P., "Performance Modeling of Location Tracking Systems," ACM Mobile Computing and Communications Review, vol. 2, pp. 24-27, July-August 1998.

[19] Baccelli, F., Machiraju, S., Veitch, D., and Bolot, J. C., "The Role of PASTA in Network Management," Proceedings of ACM SIGCOMM'06, pp. 231-242, September 2006.

[20] Nelson, R., Probability, Stochastic Processes, and Queueing Theory. Springer-Verlag, 1995.

[21] Lin, Y.-B. and Yang, S.-R., "A Mobility Management Strategy for GPRS," IEEE Transactions on Wireless Communications, vol. 2, pp. 1178-1188, November 2003.

[22] Hung, H.-N., Lee, P.-C., and Lin, Y.-B., "Random Number Generation for Excess Life of Mobile User Residence Time," IEEE Transactions on Vehicular Technology, vol. 55, pp. 1045-1050, May 2006.

[23] Khan, F. and Zeghlache, D., "Effect of Cell Residence Time Distribution on The Performance of Cellular Mobile Networks," Proceedings of IEEE Vehicular Technology Conference, pp. 949-953, May 1997.

[24] Fischer, M., Gross, D., Masi, D., and Shortle, J., "Analyzing The Waiting Time Process in Internet Queueing Systems with The Transform Approximation Method," The Telecommunications Review, vol. 12, pp. 21-32, 2001.

\section{BIOGRAPHY}

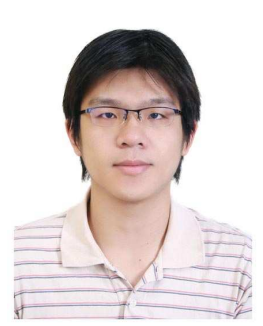

Huai-Lei Fu received his bachelor and master degrees in Computer Science and Information Engineering from Tatung University and Yuan Ze University in 2005 and 2007, respectively. Since September 2007, he enrolled his Ph.D. program in the Department of Computer Science and Information Engineering (CSIE), National Taiwan University. Now, he is a Ph.D. candidate. His research interests include machine-to-machine communications network, multicast and broadcast service, mobility management, performance modeling, and wireless sensor network. He has received the YZU Academic Silver Medal Award in 2006, the YZU School Work Silver Medal Award in 2006, the Y. Z. Hsu Scholarship Award in 2006 and 2007, and the NTU Outstanding Student Award (Academic Category) in 2009. He is an IEEE student member.

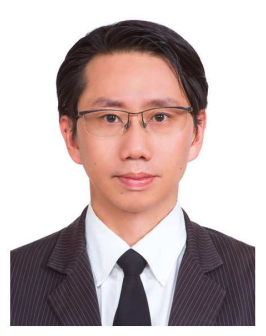

Phone Lin is Professor in National Taiwan University (NTU), holding professorship in the Department of CSIE, Graduate Institute of Networking and Multimedia, Telecommunications Research Center, and Optoelectronic Biomedicine Center. Lin serves on the editorial boards of IEEE Trans. on Vehicular Technology, IEEE Wireless Communications Magazine, ACM/Springer Wireless Networks, Computer Networks, Wireless Communications and Mobile Computing, Security and Communication Networks, IEEE/KICS Journal of Communications and Networks, and Journal of Wireless Mobile Network, Ubiquitous Computing and Applications. He is Guest Editor of IEEE Wireless Communications Magazine and ACM/Springer Mobile Networks and Applications. He has also been involved in several prestigious conferences, such as holding the Technical Program Chair of WPMC 2012. Lin has received numerous research awards, including Junior Researcher Award of Academia Sinica in 2010, Ten Outstanding Young Persons Award of Taiwan in 2009, Best Young Researcher of IEEE ComSoc Asia-Pacific Young Researcher Award in 2007, Youth Engineer Award of the Chinese Institute of Electrical Engineering in 2006, Wu Ta You Memorial Award of NSC in 2005, Fu Suu-Nien Award of NTU in 2005, Research Award for Young Researchers of Pan Wen-Yuan Foundation in 2004, and K. T. Li Young Researcher Award honored by ACM Taipei Chapter in 2004. Lin is IEEE Senior Member and ACM Member. He received his BSCSIE and Ph.D. degrees from National Chiao Tung University (NCTU) in 1996 and 2001, respectively. Lin's email and website address are plin@csie.ntu.edu.tw and http://www.csie.ntu.edu.tw/ plin/, respectively. 


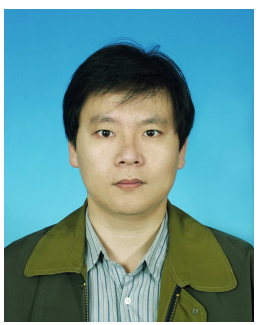

Yi-Bing Lin is Vice President and Lifetime Chair professor of National Chiao Tung University (NCTU). He serves on the editorial board of IEEE Trans. on Vehicular Technology. He is General or Program Chair for prestigious conferences including ACM MobiCom 2002. He is Guest Editor for several journals including IEEE Transactions on Computers. Lin is the author of the books Wireless and Mobile Network Architecture (Wiley, 2001), Wireless and Mobile All-IP Networks (John Wiley, 2005), and Charging for Mobile All-IP Telecommunications (Wiley, 2008). Lin received numerous research awards including 2005 NSC Distinguished Researcher, 2006 Academic Award of Ministry of Education and 2008 Award for Outstanding contributions in Science and Technology, Executive Yuen, 2011 National Chair Award, and TWAS Prize in Engineering Sciences, 2011 (The Academy of Sciences for the Developing World). He is in the advisory boards or the review boards of various government organizations including Ministry of Economic Affairs, Ministry of Education, Ministry of Transportation and Communications, and National Science Council. He is a member of board of directors, Chunghwa Telecom. Lin is AAAS Fellow, ACM Fellow, IEEE Fellow, and IET Fellow. 